Meta

Journal des traducteurs

Translators' Journal

\title{
Le DEAF en tant que dictionnaire diachronique : problèmes théoriques et pratiques
}

\section{Kurt Baldinger}

Volume 18, numéro 1-2, mars 1973

Actes du deuxième colloque international de linguistique et de traduction. Montréal, 4-7 octobre 1972

URI : https://id.erudit.org/iderudit/001953ar

DOI : https://doi.org/10.7202/001953ar

Aller au sommaire du numéro

Éditeur(s)

Les Presses de l'Université de Montréal

ISSN

0026-0452 (imprimé)

1492-1421 (numérique)

Découvrir la revue

Citer cet article

Baldinger, K. (1973). Le DEAF en tant que dictionnaire diachronique :

problèmes théoriques et pratiques. Meta, 18(1-2), 61-85.

https://doi.org/10.7202/001953ar 


\title{
Le DEAF en tant que dictionnaire diachronique
}

\author{
PROBLÈMES THÉORIQUES ET PRATIQUES
}

\section{LE DICTIONNAIRE DIACHRONIQUE (GÉNÉTIQUE)}

\section{DANS LE CADRE DE LA TYPOLOGIE DES DICTIONNAIRES}

Les dictionnaires organisent le lexique en constituant des macrosctructures sur la base des unités des rangs 1 à 4 ou même 5 d'après la hiérarchie établie par Heger ${ }^{1}$. Sur le plan synchronique, deux points de départ sont possibles : la forme de l'expression ou un sémème faisant partie d'une ou de plusieurs formes $\mathrm{du}$ contenu. Ce double aspect reflète la vieille querelle entre les partisans du dictionnaire alphabétique et ceux du dictionnaire conceptuel, entre la sémasiologie et l'onomasiologie - querelle superflue puisque la bipolarité du signe linguistique est irréductible; ces deux types de dictionnaires répondent à deux questions différentes : «Qu'est-ce que ce mot signifie ?» d'une part et, d'autre part : «De quel(s) mot(s) dispose la langue pour exprimer telle ou telle idée ?». On peut illustrer cette situation de base à l'aide du trapèze de Heger ${ }^{2}$.

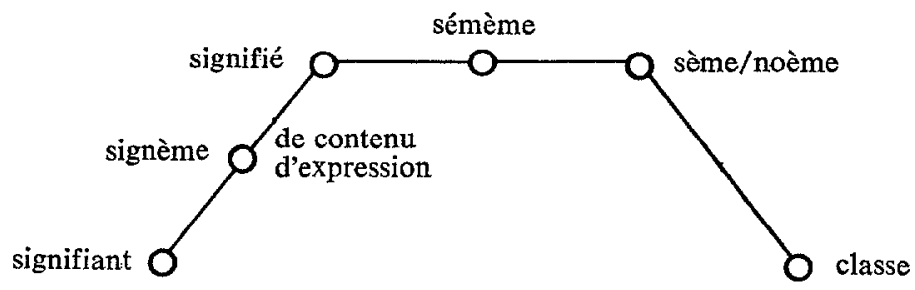

Le dictionnaire alphabétique ${ }^{3}$ ou sémasiologique est une des multiples possibilités d'organiser le lexique (les signèmes autosémiques des rangs inférieurs) d'après les relations sur le plan de la forme de l'expression; le dictionnaire conceptuel ou onomasiologique range les mêmes signèmes d'après leurs relations

1. Klaus Heger, Monem, Wort und Satz, Tübingen, Niemeyer, 1971.

2. Ibid., p. 33 ; et Klaus Heger, "La sémantique et la dichotomie de langue et parole *, TraLiLi, vol. VII, no 1, p. 47-111 (spéc. p. 69 et p. 75).

3. a L'ordre alphabétique, le plus trivial et le moins rêvé * (préface, p. Xxvil du Ménage, éd. 1750). 
sur le plan du contenu ${ }^{4}$. Le dictionnaire alphabétique détruit les rapports onomasiologiques; le dictionnaire conceptuel, les rapports sémasiologiques. Toutes les organisations intermédiaires (p.ex. : le dictionnaire analogique) ne sont en fait que des combinaisons des deux principes de base.

Le dictionnaire diachronique, le seul qui nous intéresse dans le cadre de cette communication, ajoute à ces deux principes une troisième dimension, la perspective chronologique et par conséquent génétique. Son unité de base (du côté sémasiologique) est la famille de mots plutôt que le mot isolé. La famille de mots se justifie uniquement par une origine commune, c'est-à-dire de façon génétique et non de façon sémasiologique ou onomasiologique (la définition de famille de mots sur le plan synchronique serait très différente ; cp. : la conception de l'homonymie sur les deux plans).

\section{LA FAMILLE DE MOTS - UNITÉ DE BASE DU DICTIONNAIRE GÉNÉTIQUE}

Il est évident que la famille de mots au sens génétique pose des problèmes de délimitation. Plus nous remontons dans le temps, plus la famille gagne en ampleur (les cousines au sens large remontent jusqu'à Ève !).

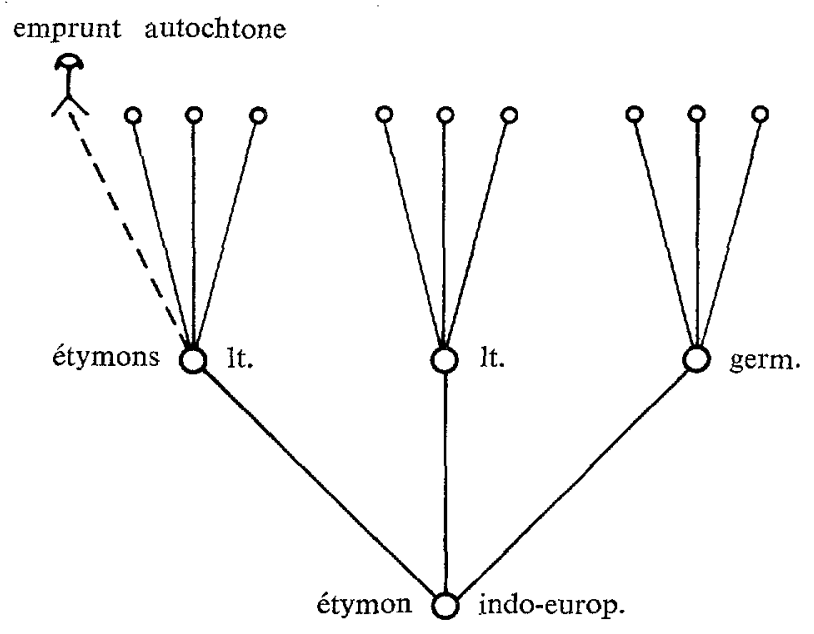

L'auteur du dictionnaire diachronique choisit lui-même son point de départ. Quant aux langues romanes, on s'est habitué à choisir, pour le point de départ, le latin (situation privilégiée) $)^{5}$.

La décision entre emprunt et dérivé autochtone n'est pas toujours facile, mais la difficulté ne consiste pas à distinguer les deux notions, qui sont tout à fait

4. Pour l'histoire de ces deux principes, voir B. Quemada, les Dictionnaires du français moderne, Paris, Didier, 1968, notamment le chapitre II de la $2^{\mathrm{e}}$ partie : « Le classement des adresses $\triangle$, p. 321-389.

5. Voir à ce sujet Alain Rey, «Le dictionnaire étymologique de W. von Wartburg : structures d'une description diachronique du lexique », Langue française, $\mathrm{n}^{\circ} 10$, mai 1971, p. 83-105 (notamment : La notion de "famille" en étymologie, p. 84ss., et L'étymon et l'unité de traitement lexicographique, p. 88ss.). 
claires ; la difficulté est d'ordre pratique, non théorique. Ainsi, le premier exemple du fr. généreux se trouve, d'après tous les manuels, depuis le Dictionnaire général (DG), dans la Vieille [Vetula] de Jean Lefevre (ca.1370). En réalité, la première attestation du sens moderne de généreux ne date que du $16^{\mathrm{e}} \mathrm{s}$. Il n'y a qu'à regarder le passage de Jean Lefevre pour s'en rendre compte :

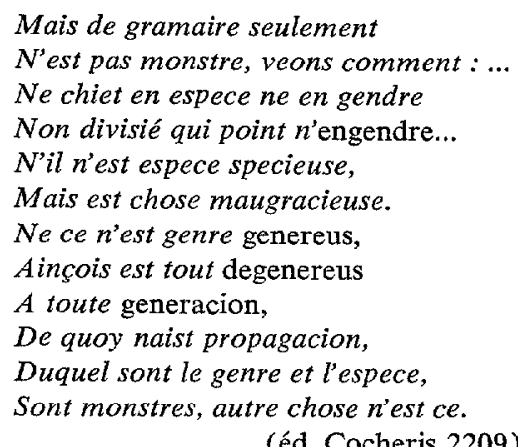

Gdf. cite pourtant cet exemple sous la déf. « qui montre de nobles sentiments »; en réalité, il faut définir "qui est capable d'engendrer (au sens fig., en parlant de la grammaire) », ce qui déplace le problème étymologique. Bien qu'il s'agisse prob. d'un emprunt à Ovide (v. generosus ThesLL 6, 1800), le sens du mot se rattachait au $14^{\mathrm{e}} \mathrm{s}$. étroitement à l'afr. generer. Il y a là des liens étymologiques à la fois vertical et horizontal.

\section{LES SOURCES}

Un dictionnaire diachronique peut se baser sur quatre catégories de sources différentes. Elles constituent toutes à la fois des sources d'informations et d'erreurs, c'est-à-dire des sources pleines de pièges; je me limiterai à quelques exemples témoins pour chaque catégorie.

\section{A. LES LEXIQUES ET LES GLOSSAIRES FAITS PAR DES CONTEMPORAINS}

"Les dictionnaires sont des plagiats par ordre alphabétique », avait constaté Charles Nodier ${ }^{6}$, et il n'était pas très loin de la vérité. J'ai montré ailleurs le mécanisme de ces transferts de dictionnaire en dictionnaire ${ }^{7}$. Souvent, les dictionnaires dits «contemporains » découvrent et admettent un mot ou une acception avec un retard considérable (parfois de quelques siècles!) ; mais dès qu'il est admis, ils ne le lâcheront plus, même s'il est mort depuis longtemps ${ }^{8}$. Il faut

6. Cité par Sachs, 1875 , p. XII.

7. Kurt Baldinger, "Autour du Französisches Etymologisches Wörterbuch (FEW). Considérations critiques sur les dictionnaires français, Aalma 1380 - Larousse 1949 ", Revista portuguesa de filologia, $\mathrm{n}^{\circ} 4,1951$, p. 342-373; v. aussi mon article "Les gaffes des lexicographes * (à paraître dans les Mélanges Lapesa) et l'ouvrage plus général de Bernard Quemada, Des dictionnaires du français moderne, 1539-1863, Paris, Didier, 1968, 683 p. (cité Quemada).

8. V. p.ex. : dénombrement, t. de jurispr. : attesté dans des textes en 1329 (Gdf), 1376 (Delb. Rec., DG), 1384 (Littré S), 1403-1581 (Gdf), il fait son entrée dans les dict. grâce à Rich 1680 ; comme t. de la jurispr. de l'Ancien Régime il devient t. hist. depuis la Révolution, mais certains dict. du $19 \mathrm{e}$ siècle continuent à le donner sans aucune remarque restrictive (Boiste 1829, Mozin 1811-1842, Land 1851, Lar 1870 et encore Lar 1929 ; l'Ac 1835 le qualifie de « vieux ", de même Besch 1845). 
donc compter avec un décalage temporel souvent important entre la réalité de l'usage et son reflet dans les dictionnaires «contemporains ». II arrive même que ceux-ci se copient mal - ce qui fait naître des mots fantômes :

mauge f. " tuyau goudronné qui reçoit de la pompe l'eau qu'on en fait sortir» se trouve dans les dict. fr. de Fur 1701 jusqu'au Lar 1873. Furetière le cite d'après un dict. de Pomey qui contient beaucoup de mots dialectaux du Sud-Est; mauge doit être une faute d'impression pour mange, forme méridionale et frpr. correspondant au fr. manche < MANICA (FEW 6 1, 209a et n 29). Le mot fantôme mauge a donc "vécu » pendant près de deux siècles dans les dictionnaires.

lièvre de beaupré " saisine de beaupré, tour de corde» (Lar 1931), enregistré sous lièvre comme t. de marine jusqu'au Lar 1931, doit sa naissance à une mauvaise lecture de lievre $=$ lieüre $=$ liûre dans Trév $1771(<$ LIGATURA, FEW 5,321 et n 10). Cette signification fantôme est donnée par presque tous les dictionnaires du $19^{e} \mathrm{~s}$., sauf par Littré (soit dit en son honneur).

Il arrive également aux dict. de sauter une ligne ou un passage en copiant leur modèle, ce qui a de curieuses conséquences : on «chante les bibliothèques », p.ex., dans les Trévoux ${ }^{9}$ ! En voici un exemple nouveau :

étoile matinière « qui travaille dès le matin »! Landais 1851. Cet ex. devient compréhensible seulement si l'on consulte Boiste 1829 : $1^{\circ}$ étoile matinière (vieux, d'après Gattel), $2^{\circ}$ matinier « qui travaille dès le matin " (cite Rouband = Roubaud, auteur de la fín du $18^{\mathrm{e}} \mathrm{s}$ ) ! Landais a sauté de $1^{\prime}$ expression $1^{\circ}$ à la définition de $2^{\circ}$ (v. FEW $6^{1}, 538$ a et n. 29).

L'ex. de moufle est particulièrement significatif pour les déformations successives d'une définition :

moufle "sorte de petit four mobile qui reçoit les matières destinées à la coupellation ( $t$. d'essayeur) 》 Enc 1765. Boiste 1803 abrège en efour mobile ». Boiste 1829, ayant lu " tour mobile", accroche à ces deux mots un complément d'explication qui les rattache à un autre sens de moufle, celui de "manchon de bois dont on entoure la tige du fer à souder »: "tour mobile de bois creux pour saisir la tige d'un fer chaud "! (cp. FEW 16,576a) 10 .

On trouve ce genre de confusion jusque dans les glossaires dialectaux : Brun, dans son livre le Français de Marseille (1931), définit il fait mauvais par "être pauvre », définition incompréhensible si l'on ne consulte pas les dict. fr. depuis Fur 1690 qui donnent l'expression il fait mauvais avec la déf. « il est désagréable, pénible » et ajoutent comme exemple il fait mauvais être pauvre!

\section{B. LES LEXIQUES ET LES GLOSSAIRES HISTORIQUES}

Ces lexiques ou glossaires peuvent concerner l'ensemble de la langue (FEW), une certaine époque (Huguet; TL; Gdf), certains auteurs (Keller, Wace) ou

9. V. mon article cité à la note $7, R P F, \mathrm{n}^{\mathrm{0}} 4,1951$.

10. Je dois cet exemple à l'amabilité de Walter Lacher qui travaille depuis de longues années avec les dict. fr. pour le FEW. Voici deux autres ex. : Grandes maisons se font par petite cuisine «c'est par l'économie que l'on devient riche 》 (DG, Lar 1907; Lar 1923 ; Lar 1931) est transformé en grandes maisons ne font pas petite cuisine dans Lar 1949 qui garde pourtant la définition, ce qui crée un contresens. Frm. nigoteaux m.pl. "quarts de tuile placés le long d'un solin ou d'une tuilée » (dep. Corn 1694), dim. de nigaud (<NICODEMUS), métaph. (propr. "petit nigaud"), est mal copié par Rich 1732 et 1759 qui écrivent nicoteux (tout en le rangeant alphabétiquement sous nigot-). Sous cette forme le mot continue à « vivre » dep. Enc 1765 jusqu'au Lar 1874. EncMA 2,72 en fait, par une seconde faute d'impression, rigoteau, forme copiée par Lar 1875 ! Véritable danse macabre de ghost words. 
certains textes (glossaires des éditions). Voici une ébauche de classification des pièges ou sources d'erreur :

\section{1. Étymologies fausses ou doubles}

Les étymologies fausses dépendent évidemment du progrès de la science. Si l'on compare Ménage et le FEW, il est impossible de nier un progrès presque miraculeux. Mais le FEW lui-même n'est pas exempt d'erreurs et de pièges étymologiques :

mfr. loquebaut " homme qui fait l'important " est expliqué comme un composé du lt. LOQUI et de l'abfrq. *BALD « courageux; impertinent » sous LOQUT (FEW 5,423a) et sous *BALD (FEW 15,31b), mais comme un composé de l'ags. loc. " loquet » et du flam. bout « boulon » sous LOC (FEW 16,474b) 11 , étymologies valables uniquement pour son homonyme aflandr. loquebaut * loquet "!

11 faut pourtant dire qu'il est rare que deux éléments d'un même mot composé soient expliqués par deux étymologies différentes.

\section{Mots fantômes, formes erronées}

Il faut en distinguer différentes catégories :

a) Mots fantômes ou formes erronées des éditions qui passent directement ou à travers les glossaires dans les manuels :

afr. gart mangier "lieu pour conserver les aliments" (ca. 1270, FEW 61,172a sous MANDUCARE, article rédigé par $T$. Reinhard), est donné comme première attestation de afr. mfr. garde-mangier (1286-1487). Gart-mangier se trouve dans l'éd. Méon du Roman de Renart (v. 15244), d'où le mot a passé dans Lacurne et dans Littré ; du Littré au FEW. En réalité, il faut lire quart \& le quatrième $*$ !

Renart, qui tant aime gelines,

De .II. chapons se rest dignez.

Puis est ou cloitre retornez.

Les.III. menja que nus nel sot;

Au quart manger iluoc passot

Un frere qui bien aparçut

Que Renart li rous les deçut.

(éd. Roques 8752)

(éd. Martin VI 1494 et les var. t. III p. 237 : gart n'existe dans aucun ms.)

b) Reconstructions fausses. - Les fausses reconstructions sont assez fréquentes, surtout pour l'époque de l'afr. Quelques exemples :

creon $\mathrm{m}$. * monde " chez Wace ; cette interprétation est un " péché de jeunesse » de $\mathrm{H}$. E. Keller dans son (excellente) étude descriptive sur le voc. de Wace, p. 29 (A toi Sire, graces en rent... Qui vis et regnes ce creon); ce creon " nous croyons", ce qui demande une virgule après regnes.

agarer, verbe donné par Gdf et Tobler-Lommatzsch (TL 1,206) est un fantôme reconstruit sur l'impératif abrégé agar! pour agarde! < agarder (*WARDôN) ; rectifié dans le FEW $17,524 \mathrm{n} 17$.

gache (et var.) * esp. de drap de laine " est un fantôme reconstruit sur le pl. gaches par Gdf (d'où aussi dans le FEW 17,541a), qu'il faut lire gachés. Le vrai sing. est gachet (-ITTU).

11. Les données des deux articles se complètent. 
aflandr. warger, etc. " observer " FEW 17,522b représenterait, d'après Wartburg, un type ancien *WARDICARE ; en réalité c'est une fausse reconstruction à partir des formes du subj. prés. garge, etc. (l'infinitif est garder) ${ }^{12}$.

c) Pastiches. - Les pastiches, fréquents en latin médiéval, sont peu connus des romanistes. Nous venons de découvrir un cas navrant : en 1832, un jeune homme de 28 ans, Charles-Joseph Richelet, s'est amusé à fabriquer et à publier trois textes en anc. fr. : Li molnier de Nemox, Du Baro mors et vis et Li neps del pastur, textes qu'il prétendait dater des $\mathrm{XI}^{\mathrm{e}}$ et $\mathrm{XII}^{\mathrm{e}} \mathrm{s}$. Godefroy les a dépouillés (en relevant au moins dix mots avec onze passages); à travers Gdf, six de ces mots ont passé dans le FEW (touzeter «faire la cour à une jeune fille», raisiner «boire du vin», teüement «tacitement», simploiant « simple, doux», riblette «tranche mince de lard $»$, raffard $\ll$ railleur $»)^{13}$.

\section{Définitions fausses ou doubles}

Les dict. et les gloss. fourmillent de définitions fausses dues à une mauvaise interprétation des textes (le Gdf en est particulièrement riche) ; il serait superflu de nous y attarder longuement (v. les nombreux cas rectifiés dans le DEAF). Qu'il me soit permis toutefois d'en citer au moins deux exemples témoins :

aflandr. rengrangement (texte de 1478 , Gdf $7,33 \mathrm{c}$ ) est défini * réparation " par Gdf. En réalité le mot se rattache à GRANDIS et signifie "agrandissement "(FEW 4,690a et n 4);

et le bel exemple de

mfr. cauresse, attesté chez Martin Lefranc et défini « sorcière » (!) par Gdf :

Sur crapaulx et raines, caurresses

Raemplies d'art dyabolique

En réalité, il faut supprimer la virgule et définir raine cauresse « rainette». Cauresse < CORYLUS « noisetier» + ARICIA (la rainette aime à se tenir sous les noisetiers), FEW 2,124lb et $\mathbf{n} 6$. de textes :

De même, il faut se méfier quelquefois des glossaires accompagnant les éd.

meure s.f. est défini «Moor » ( = "marais ") mais l'éditeur Gennrich voulait sans doute écrire «Mohr» (= "maure ") dans le gloss. de la Dame à la Lycorne (v. 1467). Le mot se trouve dans la locution plus noirs que meure et vient du lt. MORUM " mûre » (FEW 63,153a et n 55) ${ }^{14}$.

Les définitions «doubles» sont bien moins fréquentes (elles ne se trouvent que dans des ouvrages aussi compliqués que le FEW, et même là elles sont heureusement rares) :

afr. garnesture, dans un même passage de Fouke (agn. déb. 14e s.), est défini dans le FEW par " garnison » $(17,529 \mathrm{~b}$ et $530 \mathrm{a}$; cité deux fois par erreur) et par " provisions, ressource [ [déf. fausse ] (à un troisième endroit du même article 17,531b).

12. Kurt Baldinger, "Afr. garger - les deux vies d'un mot fantôme " (à paraître dans les Mélanges Lecoy).

13. V. notre article "Trois plagiats et leurs victimes en lexicologie " (à paraître dans les Mélanges Imbs, vol. des TraLiLi de Strasbourg).

14. Autre exemple : c'est bien employé est défini " c'est une bonne affaire, une chose profitable » par Cressot dans son gloss. des Quinze Joyes de mariage (III, 24, 14). Wartburg le corrige dans le FEW, 4,594a ( c'est bien fait, il l'a mérité »). Rychner dans son éd. de 1963 définit de façon correcte "vous mériteriez » (III, 88). 


\section{Erreurs dues à l'homonymie entre deux langues}

Les ex. en sont rares, mais ils sont d'autant plus amusants. Hubschmid en a découvert un à temps en lisant les épreuves d'un livre de Lahovary qui, interprétant la définition "auge » comme s'il s'agissait d'un mot allemand, l'avait traduite par « œil ». En réalité il s'agissait du fr. auge!

Mme Huyghe, rédactrice au DEAF, vient de découvrir un cas analogue dans le FEW : dans l'article abfrq. *GARD, Wartburg cite afr. jarderie, attesté dans Maurice de Sully, qu'il définit par «tares» (FEW 16,16b); tares est en effet la définition du glossaire, mais c'est une définition en anglais et le mot signifie «ivraie »; aussi faut-il enlever ce mot de cette famille et le rattacher à une famille rangée parmi les étymologies inconnues (FEW 21,143) où, en effet, jarderie « ivraie » est déjà enregistré ${ }^{15}$ !

\section{LA LITTÉRATURE SECONDAIRE}

La littérature secondaire (p.ex. : études littéraires ou linguistiques sur des textes, mots, etc.) traitant soit des aspects généraux soit des détails est d'une aide précieuse pour le rédacteur d'un dictionnaire historique. Il se trouvera souvent confronté avec toutes les explications imaginables et même inimaginables. Il risque, souvent, en tout cas, d'être entraîné sur une fausse piste. Je me passe d'exemples.

\section{LES TEXTES}

Les textes constituent, sans aucun doute, la source la plus directe et la mine la plus riche. $\mathrm{Si}$, pour un dictionnaire de la langue médiévale, les dictionnaires de l'époque (ci-dessus $\mathrm{A}$ ) ne créent guère de problèmes puisqu'ils sont pratiquement inexistants ${ }^{16}$, les textes et leur interprétation posent une infinité de problèmes. Ce sont là les problèmes essentiels à résoudre par le dictionnaire. $\mathrm{Du}$ travail philologique consciencieux de tous les jours dépend la qualité du dictionnaire. Là encore, les pièges sont nombreux.

\section{Les fautes de copistes et d'éditeurs}

Les fautes des copistes et des éditeurs sont la source de nombreux mots fantômes et de définitions erronées :

15. Je remarque en passant que TL, profitant de la polysémie d'un mot allemand, réunit assez souvent deux sens différents d'un mot afr. : ainsi, sous porgarder, il définit par nach etwas schauen aussi bien les exemples comme mes plantes purguarderunt (PsCambr $55,6)=$ "surveiller pour protéger ", que ceux du type Par soliers et par voutez l'ont quis et pourgardé (Doon 180) = "chercher "! Sous regarder (TL 8,598), " nachsehen " est pris à la fois pour "prendre en considération" (Rem. Am. 488) et pour * lire une lettre pour connaître son contenu» $\left(\mathrm{RCy}^{2} \mathrm{3200}^{2}\right.$ ). Sous regäaignier (TL 8,592), zurïckgewinnen recouvre les significations "gagner ce qu'on avait perdu (p.ex. de l'argent) s et "reprendre (une terre, une ville, etc.) à l'annemi " (v. DEAF sub gaaignier).

16. Les glossaires (v. la collection publiée par Mario Roques) apparaissent vers 1290 (GlDouai) ; leur témoignage est souvent peu clair parce qu'ils définissent, en général, un mot polysémique latin par un mot fr. également polysémique. 
rien : un trop grand rien a repasser chez Froissart, cité par Littré sub rien, est une faute pour rieu < RIvus (ex. que je dois à M. Lacher).

entoise f. « embûche » (Gdf) ; « Schlinge * (TL) dans le Roman de Brut 13647 (d'après Le Roux de Lincy) est un mot fantôme, v. l'éd. Arnold FEW 5,746 n 1.

gaintré (gaffrez) dans la Destruction de Rome v. 711 (Gdf 4,205a; TL 4,51) est un mot fantôme ; lire saffrez (DEAF).

ganele (Gdf 4,216c) est une faute pour gavele (= javele) " monceau (de bois) »< *Gabella (DEAF).

gallesuie f. (Gdf 4,211b), à lire gallesnie < GALOXINA FEW 4,48b (DEAF).

gadué (Gdf 4,199c sans déf.), à lire gadne "blond" (= jaune) < GALBINUS (DEAF). gaaille (Gdf 4,195a), dans un fabliau, a été construit par l'éditeur; l'article du DEAF, 1971 , est à corriger.

genenelle (Gdf 4,256a), à lire genevelle « penture » < GENABELLA FEW 4,95b.

\section{2. Éditions standardisées}

Certains éditeurs « reconstruisent $»$ un texte «pur» ou « original », en modifiant les formes attestées dans les mss.; ils francisent, ils picardisent, etc. Un seul ex. : Ernest Langlois, dans son éd. du Jeu de la feuillée d'Adam de la Hale ( $2^{\mathbf{e}}$ éd. 1924, réimpr. 1968), préconise «sans scrupule» dans la préface : «je n'ai éprouvé aucun scrupule à la modifier [la graphie du ms. $P$ déjà publié] lorsqu'elle était contraire soit à la prononciation artésienne, soit aux habitudes des scribes d'Arras dans la seconde moitié du XIII ${ }^{\mathrm{e}}$ s., ou pouvait être mal interprétée, ou représentait une évolution des sons trop avancée » (p. XIX $)^{17}$.

Dans le cas du Jeu d'Adam, on aura donc recours à une édition critique plus récente (de Otto Gsell 1970). Si une édition plus satisfaisante n'existe pas, il faut mettre les lecteurs en garde dans la bibliographie critique du dictionnaire.

\section{La chronologie et les interpolations}

L'original d'un texte du Moyen Âge est rarement connu (sauf pour les doc. juridiques, etc., qui, de ce fait même, sont particulièrement importants). Les textes subissent de nombreuses transformations à travers les différents mss., et cela crée de sérieuses difficultés au linguiste. On admet normalement, comme date linguistique, la date de la composition (rarement connue de façon précise), et non la date de la copie, c'est-à-dire du manuscrit (daté de même très approximativement d'après des données paléographiques, etc.). Il faudrait, en principe, relever toujours les deux dates; nous ne le faisons pourtant qu'assez exceptionnellement pour des raisons d'économie de travail. Cette distinction chronologique devient indispensable quand il s'agit de mots ou de vers interpolés; si le cas des 70 vers interpolés dans AdHaleRob ca.1285 (entre les vers 698 et 699) n'est pas grave, puisque ces vers ont été rédigés à peine trois ans plus tard (en 1288),

17. V. aussi la Vie de saint Alexis, texte critique de Gaston Paris (1903 et 1908), devenu texte classique (réimprimé dans les Classiques fr. du Moyen Age 1911, 1917, 1921, 1925,1967 ) : "Le texte que j'ai établi repose, pour les leçons, sur la comparaison méthodique des manuscrits, et, pour les formes, sur l'étude de l'évolution historique du français » (G. Paris en 1903 ; cité d'après la réimpr. de 1967, p. VI). Mario Roques parle d'un "essai de reconstitution» (ibid.). Si ce texte est utile pour l'enseignement, il ne satisfait pas aux exigences d'un dict. historique. 
l'écart entre les deux dates peut parfois atteindre, en principe, un ou plusieurs siècles.

Le même problème se pose sur le plan géographique : un texte picard, p.ex., peut être copié par un copiste franco-italien. Le rédacteur doit tenir compte de ces problèmes dans la mesure du possible et le lecteur doit en être conscient.

La bibliographie critique accompagnant le dictionnaire devra donc avertir le lecteur de ces problèmes (auteur, date de rédaction; manuscrits; dialecte de l'auteur, ceux des copistes). La rédaction d'un «Beiheft » qui la contiendra ${ }^{18}$ est un travail immense et ingrat, qui nous réserve d'ailleurs beaucoup de surprises ${ }^{19}$; bien qu'il soit encore loin d'être parfait, ce «Beiheft » constituera un précieux instrument de travail pour le linguiste ${ }^{20}$.

\section{PROBLÈMES SÉMANTIQUES}

\section{A. INTERPRÉTATION ET SIGNIFICATION}

Je ne veux pas aborder le problème théorique de l'analyse du signifié et de la signification en soi (problème central de la sémantique) et je dois me contenter de renvoyer, à ce sujet, aux beaux articles de Josette et Alain Rey ${ }^{21}$. Le problème de la définition se pose pour tous les dictionnaires, pas seulement pour le dictionnaire diachronique ou génétique. Supposons effrontément que les problèmes théoriques de la définition soient résolus. Le rédacteur d'un dictionnaire diachronique se heurte à un problème beaucoup plus immédiat : celui de l'interprétation du texte écrit. L'auteur d'un dictionnaire contemporain a à sa disposition son propre sentiment métalinguistique et il peut le vérifier ou le corriger par celui des autres contemporains. Pour une époque du passé, cette source d'information n'existe pas. Il s'agit de découvrir une langue « inconnue ».

18. La bibliographie critique du DEAF est rédigée par Frankwalt Möhren qui y travaille depuis quatre ans; une première tranche paraîtra avec le prochain fascicule.

19. Des textes « mystérieux " (cités par Gdf p.ex.) se révèlent être des textes bien connus, p.ex. Poëme alleg. Brit. Mus. = Prophéties David (ProvDav); Loherains, que Gdf cite d'après divers mss., correspond à Hervis, GarLorr, GerbMetz, AnsMetz et Yon : Hist. univ. B.N. fr. 20125 et Est. Rog. ou Estories Rogier sont identiques et correspendent à HistAnc (Histoire ancienne jusqu'à César, dans une cinquantaine de mss.); Compos. de la s. escript. = CiNDit (Ci Nous Dit, de ca. 1320 ; éd. de Gérard Blangez, thèse, Paris, 1971, pas encore publiée) ; Helias = ChevCygne (le chevalier au cygne) ; etc.

20. Le lecteur trouvera sous un même sigle toutes les éditions, même si leurs titres diffèrent, de sorte qu'il pourra toujours vérifier s'il existe une édition plus récente (p.ex. MenReimsW $=$ Récits d'un ménestrel de Reims au $13^{e}$ s., éd. N. de Wally, Paris, 1876; MenReimsP $=$ Chronique de Rains, éd. P. Paris, Paris, $1837 ;$ MenReimsS $=$ Chronique de Flandre et des Croisades, éd. J. J. de Smet dans la coll. des Chroniques de Flandre, t. III, Bruxelles, 1856; malgré les titres divergents, il s'agit de trois éditions d'un même texte).

21. Josette Rey, "La définition lexicographique : recherches sur l'équation sémique », CahLex, no 8, 1966, p. 71-94; A. Rey, «A propos de la définition lexicographique ", CahLex, no 6, 1965, p. 67-80; "Les dictionnaires : forme et contenu ", CahLex, no 7 , 1965 , p. 65-102. Pour d'autres travaux v. mon livre Teoría semántica, Hacia una semántica moderna, Madrid, Alcalá, 1970 ; quant à l'histoire de ces problèmes, v. Bernard Quemada, les Dictionnaires du français moderne, 1539-1863, Paris, Didier, 1968, $2 \mathrm{e}$ partie, chap. III : "Les définitions ", p. 391-464; "Analyse et classement des sens », p. 365-504. 


\section{Danger d'interpréter par le frm.}

Notre connaissance du frm. est plutôt un obstacle et une source d'erreurs ! Ainsi, en analysant l'afr. garnement, j'ai eu beaucoup de difficultés à séparer les sens de «vêtement» et $d$ '« ornement de vêtement (pierres précieuses, etc.) ». Au premier moment, un texte au moins me semblait clair :

Deux, tant forment reluisent si (de Florence) garnemant

Entre lor et les pierres, dom il i avoit tant

(Florence 4174)

$\mathrm{Si}$, entre l'or et les pierres, on peut entrevoir le garnement, je pensais qu'il devait bien s'agir d'un vêtement... Heureusement, je me suis aussitôt souvenu que entre afr. avait eu des acceptions aujourd'hui perdues, p.ex. :

Enter mirra et aloen

Quasi cent liuras adonad

(Passion 347)

c'est-à-dire «myrrhe et aloès ensemble », et le sens du passage de Florence m'est apparu clairement : «ses garnements, l'or et les pierres tout ensemble reluisent... Ce passage est donc plutôt une illustration du sens que je croyais éliminé : «ornement, pierres précieuses, joyaux portés par une personne $» !$ Par conséquent, le problème n'est pas celui de distinguer les notions de «vêtement » et de «pierres précieuses, ornement », mais celui d'attribuer un passage à l'une ou à l'autre des deux notions.

\section{Danger d'interpréter d'après un "petit » contexte}

Souvent, le contexte immédiat permet plusieurs interprétations. Prenons relenquir dans le roman de Jason (ca.1460, éd. Pinkernell 1971) :

J'ay fait tout a bonne intencion pour vostre grace acquerre, vous me relenquissiez et reppudiez $(18.3,19)$.

... sitost que je fu couchié, une malvaise voulenté me prinst, qui fu telle que je relenquis vostre amour et conclus que de Medee je feroye ma dame, comme je fis $(19.1,29)$.

Le FEW nous donne les significations : $1^{\circ}$ «laisser dans la détresse, priver de soutien » SFoy, fr. « abandonner, quitter » ca.1171 - ca.1520 (FEW 10,232b) ; $2^{\circ}$ 《trahir»ca.1165 - 1520 (FEW 10,233a) ; $3^{\circ}$ «renier $1^{2}-15^{e} \mathrm{~s}$. (FEW ibid.).

Les «petits » contextes permettent d'admettre, pour le premier exemple, les trois acceptions (prob. $c$ ), pour le second, les acc. $b$ et $c$ (prob. $b$ ). Une interprétation sûre ou, au moins, plus sûre doit recourir au «grand» contexte.

\section{3. «Petit» contexte et comparaison avec d'autres manuscrits}

Un « petit» contexte induit souvent en erreur à coup sûr. Ainsi pour degaster v.n. « se gâter, être détérioré » (Gdf 2,471b), dans :

Or degaste la beauté et la coulour de mon viaire, et la grande gloire de ma beauté est cheute (Discipl. de Clergie, XXX ; Bibliophil. fr. Petri Alfonsi Disciplina clericalis, fr. Prosatext, édit. par A. Hilka et W. Söderhjelm, Helsingfors 1912, p. 37). 
Presque automatiquement le lecteur qui ne connaît que ce passage, pensera qu'une vieille femme se plaint de la perte de sa beauté sous l'effet du temps. La vérification du passage dans l'éd. critique de Hilka et Söderhjelm (1912, p. 37) montre qu'il n'en est rien. Il s'agit d'un homme mort gisant au tombeau. Le ms. K donne : Or me devaste chi tere le coulour de men viaire (la terre détériore, abîme la couleur de mon visage); le copiste du ms. B a prob. omis tout simplement la terre.

\section{Importance de la dialectologie}

Ou bien, prenons le passage suivant de Floovent 731 :

Aï! Maonmot sire, ja estes vos si ber,

Jai ne porai sans vos gresilier ne vanter.

On est tenté d'interpréter gresilier comme une forme déformée (attestée) de gasilier «faire des plaisanteries 》 (le jeu des gabs, des vantardises spirituelles, était très à la mode parmi les chevaliers), d'autant plus qu'il est accompagné de vanter! Mais le jai montre qu'il s'agit d'un texte ou d'un ms. lorrain et que porai $n^{\prime}$ 'est pas la $1^{\text {re }}$ pers., mais la $3^{\mathrm{e}}$, de sorte qu'il faut interpréter : «jamais il ne pourra plus sans vous faire de la grêle et du vent ». Le gresilier de notre passage ne vient pas de l'anord. gabb « raillerie », mais de l'abfrcq. GRISILÔN « grêler ». L'exemple prouve l'importance de la dialectologie pour l'interprétation des textes.

\section{Comparaison avec d'autres textes}

Comment interpréter le passage suivant :

Le meilleur warnement de robes part qu'il averoit au jour de sa mort (texte d'archives cité par Gdf 4,233c)?

Que partage-t-on à sa mort? les meilleurs vêtements ou le meilleur équipement? Robe peut avoir, comme garnement, les deux sens. Heureusement, d'autres textes viennent à notre aide, p.ex. le passage suivant des Digestes :

Ausint aura il l'usefruit des garnemens de la tenure (Gdf 4,233c).

Le sens de garnement est «équipement (en biens meubles) 》 dans les deux passages. L'interprétation doit donc souvent s'appuyer sur un jeu de comparaison qui remplace la conscience métalinguistique.

\section{Le problème de la « synonymie » et de la complémentarité}

Robe et garnement se retrouvent dans d'autres contextes, p.ex. dans le Livre des métiers de ca.1268:

Quiconques est taillieres de robes à Paris, et il mestaille $\mathbf{j}$ robe ou $\mathbf{j}$ garnement [...] li meffaiz doit estre veuz et regardez par les mestres [...] et se li mestres dient par leur serement que le garnement soit enpiriez par mestaillier... (éd. Depping p. 143).

Robe et garnement sont-ils ici complémentaires, comme dans d'autres contextes, et garnement signifie-t-il «chaque vêtement composant la robe » (p.ex. une robe de 4 garnemens ca.1302, Mahaut 165; DEAF garnement $8^{\circ}$ ) ? Cette interprétation est exclue puisque robe et garnement sont repris par le seul terme garnement dans la suite du passage, ce qui prouve que les deux termes sont synonymes. 
Mais chaque cas de combinaison «synonymique $»$ doit être examiné soigneusement. Le passage suivant est significatif à ce sujet :

plusurs serianz e provoz e dayes [...] dounent e gastent e manguent del blanc $[=$ lait, crême, etc.].

Comme gaster peut avoir en afr. le sens de \& manger », on est tenté d'y voir une synonymie parfaite. Mais le passage continue :

E sachet pur veyr le blanc ne seyt despendu ne gasté aylurs ke en la chose meymes de tant deyvent e ben poent respondre (Hoseb Hen L 26 [mil. 13e s. agn.]).

Ainsi, dans ce même contexte, gaster est combiné avec despendre, apparemment avec le même sens de «user, consumer, souvent sans mesure, gaspiller (attesté pour gaster de Chrestien à Trév 1752, FEW 14,204a)! Gaster, dans notre passage, signifie donc prob. «employer, user», à la fois au sens de «donner», de « manger », etc.

\section{Limites de l'interprétation}

Ces exemples doivent suffirent pour montrer à quel point le travail d'interprétation est délicat; il faut souvent un véritable travail de détective. Souvent aussi, il est impossible d'arriver à une interprétation sûre. Les chevaliers

Quant de tant riches armes esgardent la brunor,

Et $l i$ solaus qui done as armes grant luor...

(AlexParA II 1251)

《 regardent $»$ (actif) ou « voient (sans le vouloir) l'éclat métallique des armes ? Esgarder signifie normalement « regarder », mais, dans certains contextes, « voir »!

\section{B. L'INFLUENCE DE L'ÉTYMON SUR LA DÉFINITION}

Dans- les dictionnaires et dans les glossaires, la définition est souvent influencée par l'étymologie qu'on attribue, souvent à tort, au mot en question. Sous horion «coup violent», le Petit Robert renvoie à l'afr. oreillon « coup sur l'oreille». En réalité, aucun passage de l'afr. ne permet de donner cette définition de oreillon, qui se base uniquement sur un rapprochement étymologique avec oreille (FEW, 1,179b AURICULA). Il y a beaucoup de chance qu'en afr. il s'agisse en fait d'une déformation de horion par étymologie populaire (en afr. mfr. horion est attesté 13 fois, oreillon 1 fois, orillon 1 fois).

Mfr. remaux «saindoux» (orl. 1392-1400, FEW 10,242b) est défini par Gdf «suif en branches» $(6,775)$ parce qu'il le rattache à RAMUs (FEW ib. n 6 ). Afr. dunois (Perceval, ms. Montp. H $249=$ seconde continuation, écrite entre 1180 et 1205 d'après Lévy; le FEW indique Chrestien par erreur) est défini "obscur, noir, ténébreux » par Millet 41 parce qu’il le rattache au moy.angl. don, dun, donne, dunne (MED) ; Gdf le donne sans déf. et Wartburg le range parmi les mots d'origine inconnue (FEW 23,184a) :

A destre garde, s'a veu

L'uis d'une chambre overt ariere

Et a l'ostel d'une charriere

Une chose dunoise avoit

Et lorsque Perceval la voit

Cele part vient grant aleure

(Perceval, ms. Montp. H. 249 = Cont. Perc. éd. Potvin, t. 3, 106 [v. 233 orl : danois !]) 
TL définit au (el) regart de «im Hinblick auf» (à l'égard de) parce que 1'all. blicken correspond à regarder; mais, d'après les textes, la signification en est « en comparaison de ».

Afr. gastroillier, un hapax du Roman de Marque, est défini « donner à qn des coups dans le ventre » par l'éditeur Johann Alton parce qu'il rattache le mot au grec gaster «ventre . TL est plus prudent : «jem. (wie?) misshandeln»:

Ele fu aussi come demie morte, eschevelee et gastroilliee et batue (Marque A 39c3 ; TL $4,215)$.

Wartburg (FEW 14,203b et n 9) précise « chiffonner, friper, déchirer (les habits) » et l'explique comme croisement de VASTARE avec le verbe populaire fatrouiller, dér. de FARSURA (FEW 3,422); le mot vit encore avec le même sens en wallon. Cette interprétation est d'ailleurs confirmée par un passage du même texte qui décrit la même situation : Mout fu la dame chapigniee et peliciee et desciree (Marque A 38d4). Ce second exemple est doublement significatif : il montre d'une part le danger de se laisser influencer par une fausse étymologie, et d'autre part, le bénéfice que l'interprétation peut tirer de la reconnaissance des vrais rapports étymologiques (Wartburg, grâce à ces rapprochements, a donné une définition exacte, sans connaître le passage parallèle qui la confirme). Mais il va sans dire qu'il faut se méfier des définitions étymologiques à la Nicot : patouiller $\ll$ touiller avec la pate ${ }^{22} »$ !

\section{LANGUE ET PAROLE : SIGNIFICATION GÉNÉRALE ET SIGNIFICATION SPÉCIALE}

Voilà un problème particulièrement épineux. Je l'ai montré, il y a une dizaine d'années, en étudiant les champs sémasiologique et onomasiologique de l'apr. trebalh, et je suis arrivé à la conclusion que la différenciation des significations se réduit à un problème statistique. En effet, dès que je me sers d'un mot avec son acception centrale - supposons pour le moment qu'il y en a une et que nous croyons la connaître - dans une situation particulière, il prend, par ce fait même, une nuance significative particulière. La situation particulière réduit une signification plus générale en l'enrichissant de sèmes différenciateurs dus au contexte particulier. Nouvelleté est défini «péage nouveau» par Mantellier dans un ouvrage traitant de la navigation de la Loire (au $14^{\mathrm{e}}-15^{\mathrm{e}} \mathrm{s}$.), d'où ce sens spécialisé dans le FEW (7,203b). C'est ainsi que de nombreux mots deviennent des termes techniques, des termes précis de métier. Un terme précis de métier, employé, au contraire, en dehors de la situation précise de ce métier, dans un contexte plus général, perd de sa spécificité : il élargit son contenu sémantique distinctif en réduisant le nombre de ses sèmes.

Il suffit de lire, p. ex., l'article FORTIS du FEW. L'acception générale « qui a de la puissance» prend, selon les contextes, 15 significations différentes : $1^{\circ}$ stark (qui a beaucoup de vigueur physique), $2^{\circ}$ dicht (touffu, dru, épais), $3^{\circ}$ dick (grand et puissant de corps, épais de taille), $4^{\circ}$ mühsam (pénible, difficile), $5^{\circ}$ stürmisch (houleux, de la mer), $6^{\circ}$ dauerhaft (qui a une grande solidité,

22. Cité par Quemada, p. 445 (v. FEW 8,36b) ; v. aussi ibid., p. 433 (marques génétiques ou causales) et p. 442 (rapports étymologiques). 
p. ex. drap, cuir), $7^{\circ}$ hart (dur), $8^{\circ}$ lehmig (se dit des terres où l'argile domine et qui sont difficiles à labourer), $9^{\circ}$ übergewichtig (denier, monnaie) dont la valeur réelle dépasse celle qu'on leur attribue), $10^{\circ}$ militärisch stark (fortifié, facile à défendre), $11^{\circ} \mathrm{sehr}$, viel (très, beaucoup), $12^{\circ}$ alkoholhaltig (qui a beaucoup d'alcool, p. ex. vin), $13^{\circ}$ sauer (acide, aigre), $14^{\circ}$ ranzig (rance, du beurre), $15^{\circ}$ laut (parler fort parler à haute voix, etc.).

Voici quelques exemples récents notés au cours de la rédaction du DEAF. Keller, dans son vocabulaire de Wace, définit soi regarder par «se retourner », d'où, ce sens particulier dans le FEW, uniquement pour Wace (FEW 17,510b). Or, les passages cités, surtout le deuxième ( Mult se vont sovent regardant, ke Normant nes augent siwant $\gg$ Rou III 1161), montrent de façon évidente qu'il s'agit du sens «normal » de «regarder autour de soi », très souvent attesté en afr. (FEW : 12 $\mathrm{e}_{\mathrm{s}}$ - - 1315). Si je regarde autour de moi, il faut bien que je me retourne, mais cette conséquence n'est qu'accessoire aussi longtemps que le sens « regarder » reste vivant et qu'un contexte ne montre de façon évidente que l'accent s'est déplacé du « regard » vers le « mouvement du corp dans la direction opposée ». Par conséquent le DEAF, n'a pas séparé cet exemple en lui accordant une signification à part. Les «deux » significations viennent uniquement d'une analyse sémantique différente des linguistes.

Autre exemple : galet "grand caillou » se trouve dans Ambroise (agn. ca. 1195), dans le contexte suivant : «Cil pautonier se defendouent Od granz jalez qu'il aportouent. »Faut-il définir galet par « grand caillou servant de projectile »? La finalité, en effet, entre souvent dans la définition ${ }^{23}$. Mais il est évident que les galets ne servaient qu'accidentellement de projectiles, tout comme une table ne devient qu'accidentellement un siège quand je m'assieds dessus. Là encore, c'est une affaire de statistique : si je trouve beaucoup de passages, dans les textes médiévaux, qui témoignent du fait que les galets servaient de projectiles, il faut, à mon avis, distinguer cet emploi par une «marque de finalité » (selon l'expression de Quemada).

Cette réflexion est valable pour un dictionnaire synchronique (caractérisant une époque). Dans le cas d'un dictionnaire diachronique, le problème est plus complexe : un exemple même isolé, qui ne mérite, du point de vue synchronique, aucune distinction, peut devenir significatif dans une perspective historique; cet exemple peut être, sur le plan de la parole, le précurseur de ce qui, plus tard, deviendra une signification sur le plan de la langue. Le mfr. connaît en effet les expressions arc à jalets $(1478 ; 1480$ : Rabelais) et arbaleste à jalet (Brantôme). Un dictionnaire historique ou génétique devra donc tenir compte de ce fait et retenir les emplois que j'appellerais «présignificatifs» (v. DEAF sub GAL).

Le même problème se pose pour les emplois métaphoriques. Des métaphores dans la parole peuvent précéder les métaphores lexicalisées et le dict. hist. devra en tenir compte (auj., p. ex., on ne pense plus au sens concret en disant dans un congrès : cette communication est une véritable salade).

23. V. p.ex. capituler "parlementer pour traiter de la reddition d'une place" (Lavaux) et les autres exemples cités par Quemada, p. 434. 
Rappelons que le fait inverse - à savoir que les dict. synchroniques lexicalisent une métaphore de parole sans que cela corresponde à une réalité d'emploi n'est malheureusement pas rare. Lamartine, p. ex., parlait d'un vent de mort qui soufflait sur la famille royale. Lar 1876 cite vent de mort «cause, influence qui produit des morts nombreuses » avec renvoi à Lamartine, ce qui est correct. Mais à partir de Lar 1907 le renvoi à Lamartine manque de sorte que la métaphore se trouve faussement lexicalisée.

\section{RÔLE DES CITATIONS DANS LE DICTIONNAIRE HISTORIQUE}

La fameuse dispute sur la question de savoir s'il faut citer des exemples d'auteurs (les « autorités ») ou donner des exemples forgés ad hoc pour illustrer les différentes significations, dispute suscitée par les grands dictionnaires monolingues de la fin du $17^{\circ}$ s. ${ }^{24}$, ne concerne pas un dictionnaire historique puisque la conscience métalinguistique d'un sujet français du Moyen Âge ne m'est accessible qu'à travers les textes (la proposition de Sofer qui voulait faire frapper des disques en lat. vulg. pour en mieux connaître la prononciation n'a pas trouvé d'écho). Cela prive l'auteur d'un dict. hist. de la possibilité d'illustrer les significations par des exemples du genre de ceux de Palsgrave qui, en 1530, a forgé, comme exemple de l'expression a talent, la phrase ma femme m'a batu a son talent (Palsgr p. 832). Les textes, c'est-à-dire les citations, qui sont la seule source primaire, deviennent alors d'autant plus importants. Le FEW, il est vrai, a renoncé, en tant que dictionnaire linguistique, à toute citation ; Wartburg a même insisté sur ce point et $\mathrm{y}$ a vu la principale différence face aux dictionnaires philologiques tels que ToblerLommatzsch, Godefroy ou Huguet, dont le but est d'aider à lire et à interpréter les textes. Nous avons suivi son exemple dans le premier fascicule du DEAF. Mais, en cours de route, nous avons changé d'avis. Les remarques que nous avons faites sur la distinction des significations, c'est-à-dire sur l'établissement des champs sémasiologiques, mettent en évidence le fait que cette distinction est très délicate et dépend dans une large mesure des contextes. Sans aller jusqu'au bout en affirmant qu'il y a autant de significations que de contextes - position qui peut se défendre sur le plan théorique mais qui détruirait la notion de «signification » au niveau de la langue (de la même façon on pourrait atomiser la notion de «langue », elle-même) - j'admets que toute définition généralise nécessairement en négligeant les nuances, ce qui est une conséquence inévitable et fatale du processus d'abstraction ${ }^{25}$. L'ensemble des définitions qui recouvrent un champ sémasiologique ne nous offre forcément qu'un squelette qui est arbitraire dans ce sens que les fils reliant les différentes significations entre elles sont coupés. Les citations peuvent rétablir les nuances et les relations mutuelles entre les différentes significations. Elles corrigent ce que les significations ont de trop rigide et les enrichissent. Elles ajoutent, ce qui dans un dictionnaire historique n'est pas sans intérêt, des informations encyclopédiques ${ }^{26}$ qui, elles aussi, complètent la signification (ainsi la définition de regart par "contrôleur, administrateur, gouverneur », est complétée

24. Quemada, p. 539ss.

25. C'est surtout vrai pour les définitions d'un dictionnaire diachronique qui, souvent, ne peut donner que des définitions peu spécifiques (elles dépendent uniquement des contextes dont on dispose).

26. Quemada, p. 76ss., 522ss. 
par des passages tirés de documents qui en décrivent les fonctions, le nombre, leur serment, leurs problèmes, etc.).

\section{E. CLASSEMENT DES SIGNIFICATIONS}

\section{Considérations générales}

Il existe très peu de travaux théoriques sur le classement des significations. Et c'est pourtant un problème auquel aucun dictionnaire n'échappe. Quemada a raison quand il affirme : "Toute étude comparative met en évidence que chaque lexicographe original (c'est-à-dire qui ne se contente pas de reproduire des répertoires antérieurs) tend à découper la réalité [nous dirions le signifié !] d'une manière qui lui est relativement propre» (p. 474). Dans le chapitre « Hiérarchie et classement des sens » (p. 487ss.), il oppose le classement appelé logique au classement historique qui se base sur une conception généalogique (p. 499). Littré est net sur ce point! «Pendant que, dans l'article consacré à l'usage présent, les acceptions sont rigoureusement classées d'après l'ordre logique, c'est-à-dire en commençant par le sens propre et en allant aux sens de plus en plus détournés, ici [dans la partie historique] tout est rangé d'après l'ordre chronologique. Le principe de succession prévaut sur le principe de l'ordre des significations; ce qui importe, c'est de connaître comment les emplois se succèdent les uns aux autres et s'enchaînent 》 (Préf. tome I, p. vII ; Quemada 502). Il va sans dire que le dictionnaire historique ou génétique doit suivre le second principe, le principe génétique. Je laisse donc de côté le problème (certainement difficile) du classement dit "logique $»$ qui ne concerne que les dictionnaires synchroniques.

Si le principe génétique est parfaitement clair, son application l'est beaucoup moins et pose des problèmes. Tout d'abord parce que notre documentation est loin d'être exhaustive. Nous dépendons du hasard des textes écrits, du hasard des textes conservés, du hasard des textes publiés et du hasard des textes dépouillés. Bien que le lexique de l'afr. soit mieux connu que celui de n'importe quelle autre langue du Moyen Âge (peut-être à l'exception du moy. angl.), il ne faut jamais oublier que la base reste toujours lacunaire et fragile. Par conséquent, une part plus ou moins large d'hypothèse est inévitable, surtout pour certains secteurs du lexique. Si la terminologie religieuse, p. ex., nous est assez bien connue dès les premiers textes littéraires et les premières traductions des psaumes (ca. 1120) ${ }^{27}$, le langage familial et argotique, p. ex., n'apparaît que de façon sporadique à la surface des textes écrits (v. Bodel, les fabliaux, AdHaleFeuill, Rutebeuf), et la terminologie dialectale est attestée très imparfaitement à travers les scriptae régionales. Voici des indications provisoires concernant l'apparition des textes de caractère spécialisé :

Jurisprudence : La terminologie juridique n'apparaît de façon systématique qu'au cours du $13^{e} \mathrm{~s}$. (à l'exception des Lois de Guillaume, écrits vers 1150); les doc. jur. en langue vulgaire abondent seulement à partir de 1240, v. les plus Anciennes Chartes en langue fr., p.p. L. Carolus-Barré, I, Paris 1964 (la plus

27. De même, la terminologie des lapidaires nous est bien connue depuis LapidAL (ca. 1125) attribué à Philippe de Thaon. 
ancienne charte de ce recueil concernant l'Oise est de 1241) ; v aussi Lois Godefroy (Cambrai 1227 texte lat.; trad. fr. contemporaine ca. 1230); Très anc.

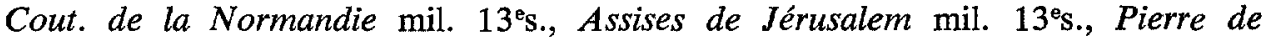
Fontaine ca. 1255, Livre de jostice et de plet ca. 1260, CoutStAmand 1265 ; EtSLouis ca. 1273 ; Britton ca. 1275 (agn.) ; CoutNormGuillT (Coutumier rimé par Guillaume Chapu) ca. 1280 ; Institutes de Justinian en vers normands ca. 1280 ; Liber Custumarum et Liber Albus (agn. à partir de 1280); CoutBeauvaisis 1283 ; Roisin fin $13^{\mathrm{e}} \mathrm{s}$., ; Anc. Cout. d'Artois ca. 1300 ; etc.

Astrologie : la Petite Philosophie éd. Trethewey 1939 (déb. 13es.); ca. 1273 Abraham Ibn Ezra, The Beginning of Wisdom en judéofr.; $2^{\mathrm{e}} \mathrm{m} .13^{\mathrm{e}} \mathrm{s}$. le Livre de Sidrac; ca. $1320 \mathrm{Li}$ Compilacions de le science des estoilles éd. Carmody 1947, etc. ; v. aussi les Compuz à partir de Philippe de Thaon (composé en 1119).

Héraldique : Un armorial fr. du mil. du XIII's. (éd. p. Paul Adam-Even, Archives héraldiques suisses $63,1949,15-22 ; 68-75 ; 115-121){ }^{28}$.

Médecine : ca. 1256 Aldebrandin de Sienne, le Livre des simples medecines (éd. par P. Dorveaux 1913), une série de recettes médicales à partir du $13^{\mathrm{e}} \mathrm{s}$. (p. ex. l'Antidotaire Nicolas $2^{\mathrm{e}} \mathrm{m} .13^{\mathrm{e}} \mathrm{s}$.), le gloss. ar. fr. de Messer Guglielmo de ca. 1300 (éd. Incichen 1972), et la Chirurgie de Henri de Mondeville (ca. 1314).

Chasse : La Chace dou Cerf (éd. Tilander $1960 ; 2^{\mathbf{e}} \mathrm{m} .13^{\mathrm{e}} \mathrm{s}$.), le Livre du roi Dancus (éd. Martin-Dairvault 1883, avant 1284 ; texte lat. éd. p. Tilander 1963), et la Vénerie de Twiti (agn. $1^{\text {er }}$ q. $14^{\mathrm{e}} \mathrm{s}$., éd. Tilander 1956).

Métiers : Règlements sur les Arts et Métiers de Paris, rédigés au XIII ${ }^{\mathfrak{e}}$ siècle et connus sous le nom du Livre des métiers d'Étienne Boileau, p.p. G.-B. Depping, 1837 (vers 1268).

Économie et agriculture : La terminologie de l'économie ménagère et de l'agriculture (agn. ca. 1240 les Regles de Robert Grosseteste, mil. 13 ${ }^{\mathrm{e}} \mathrm{s}$. Husbandry anon., $2^{\mathrm{e}} \mathrm{m} .13^{\mathrm{e}} \mathrm{s}$. Seneschaucie et Henley's Husbandry) ${ }^{29}$.

Navigation et pêche : Black Book of the Admiralty (agn. à partir de 1291), Cout. d'Oléron 1345.

Inventaires et comptes (riches en termes de meubles, d'ustensiles, d'étoffes, etc.) à partir de 1276 (Comptes de la ville d'Ypres 1267-1329 p.p. DesMarez et DeSagher, 1909) ; Mahaut 1302-1329; Comptes Royaux p.p. Fawtier ; Cpt Fleuri 1317 ; InvClemD 1328 ; CptTadD 1342, etc.

Cuisine : La terminologie de la cuisine (très riche en termes de poissons, de gibier, de plantes et d'assaisonnements ) fait son apparition systématique seulement avec les Enseingnemenz qui enseingnent a apareillier toutes manieres de viandes (éd. Lozinski 1933) de la fin du $13^{\mathrm{e}_{\mathrm{s}}}$. et le Viandier valaisan de ca. 1300

28. V. un bonne bibliographie dans le livre récent de Gerard J. Brault, Early Blazon, Heraldic Terminology in the Twelfth and Thirteenth Centuries with Special Reference to Arthurian Literature, Oxford, Clarendon Press, 1972.

29. Ces textes sont tous édités dans Walter of Henley's Husbandry p.p. Elisabeth Lamond, Londres, 1890 ; v. aussi une autre version de la Husbandry p.p. Louis Lacour, Traité d'économie rurale, BEC 17e ann., t. II, 4e sér., Paris, 1856, p. 123-141 et 367-381. V. aussi Britton et Fleta (ce dernier mlt. fin $13^{\mathbf{e}} \mathbf{s}$.). 
(éd. Aebischer) ; le «Fabliau» de Cocagne éd. par V. Väänänen $\left(2^{\mathrm{e}} \mathrm{m} .13^{\mathrm{e}}\right.$, Neuphil. Mitt. 48, 3-36) et la Bataille de carême et de charnage éd. par Lozinski 1933 $\left(2^{\mathrm{e}} \mathrm{m} \cdot 13^{\mathrm{e}} \mathrm{s}\right.$.). De grande importance aussi les Deux traités inédits d'art culinaire médiéval [en mlt.] (p.p. Marianne Mulon, Bull. phil. et hist. année 1968, Paris 1971, p. 369-435 (《remontant au moins au XIV"s. »).

Grammaire : La terminologie grammaticale apparaît ca. 1250 avec le Petit traité de conjugaison française contenu dans le ms. Cambr. R. 3.56 (p.p. P. Meyer, R 32, 66-67; ms. du mil. du 13's., agn.), ca. 1260 Li Abecés por ekivoche et li significations des lettres par Huon de Cambrai (éd. Langfors 1913), ca. 1290 Le Traité de Walter de Bibbesworth sur la langue fr. (éd. A. Owen 1929), $1^{\mathrm{re}} \mathrm{m} .14^{\mathrm{e}} \mathrm{s}$. Orthografia gallica (éd. J. Stürzinger, Heilbronn 1884; agn.), agn. 1396 Manière de Langage par Coyfurelly (p.p. J. Gessler, Louvain 1934), etc.

Les premières dates - toujours provisoires, hélas! et imparfaites, même si elles étaient exhaustives - ne sont donc pas en mesure de nous donner une chronologie absolue; le rédacteur doit tenir compte des lacunes de la documentation pour les apprécier à leur juste valeur avant de reconstruire l'histoire génétique des significations qui gardera toujours une part de provisoire.

Comme exemple d'une telle reconstruction je vous prie de lire le commentaire étymologique et génétique du verbe garnir (article du DEAF que je viens de rédiger) qui, en afr., est beaucoup plus riche en acceptions, et d'examiner le tableau synoptique ci-joint qui inclut les dérivés :

GARNIR v. [D'un germ. *WARNJ $A N, * W A R(A) N \bar{O} J A N$ \& prendre garde à qch. " qui appartient à la famille indo-eur. *uer - " apercevoir, faire attention » (cp. It. vereri " avoir une crainte respectueuse pour, révérer " asax. waron, aangl. warian, angl. beware, all. (be-)wahren) ; la forme en -jan, base du fr. -ir, est bien attestée en aangl. : warenian, wearnian, warnian (cette forme attestée p.ex. en 1023, $O E D$ ) avec les sens " to take need, be on one's guard, beware", " to make aware, to put on one's guard» (attesté dep. ca. 1000), « to put (a person) on his guard, to caution against some person or thing as dangerous " (dep. 1225), etc. (OED sub warn 1 et garnish; il paraît avoir eu selon le $O E D$ une certaine confusion en germ. et en aangl. avec un verbe homonyme *warnjan " to ward off, prevent, refuse ", asax. wernjan, aangl. wiernan, etc.). Sur le continent sont attestés aha. uuarnôn "avertir amicalement, protéger; v. pron. se garder » chez Notker, warnön " prospicere, munire, admonere, instruere, attendere " (Graff I 949 ; Massmann), gawarnôn "providere, instruere, munire, contestari ", warnunga f. « munimentum, defensio, praesidium», furiwarna « praeparatio», mha. mba. all. warnen " avertir (d'un danger) ", mnéerl. waernen «id. ; pourvoir de qch., équiper »; en mha. warnen avait aussi les sens de "préparer, équiper, protéger» qu'on retrouve en afr. En afr. garnir présente, en effet, différents sens attestés déjà dans les langues germaniques. Le point de départ est sûrement " avertir (d'un danger) 》 $\left(1^{\circ}\right)$, d'où " exhorter (qn à faire qch.) » $\left(2^{\circ}\right.$; sens rare en fr. et prob. influencé par l'anglais) et, avec perte de la notion de danger, « instruire, informer $\left(3^{\circ} ; \mathrm{cp}\right.$. en angl. le même sens dep. ca. 1205). Du sens « se tenir sur ses gardes * $\left(4^{\circ}\right)$ qui se rattache au sens $1^{\circ}$ on passe à * occuper, faire occuper (un lieu pour le défendre) " $\left(5^{\circ}\right.$; cp. garnison), terme par excellence des chansons de geste, tout comme estre garni de " muni et armé de » et sei garnir "se préparer pour le combat, s'armer» $\left(6^{\circ}\right)$, d'où, de façon plutôt sporadique, en quittant rarement le domaine militaire, le sens plus général « se disposer à, se préparer à " $\left(7^{\circ}\right)$, d'où aussi, dès le milieu du $12^{\mathrm{e}} \mathrm{s}$, en quittant cette fois-ci très souvent le domaine militaire, garni de "prêt à " $\left(8^{\circ}\right.$; cp. all. gerüstet "armé » > "prêt $)$. Le sens " pourvoir de " (concrétisé déjà dans $5^{\circ}$ ) apparaît de façon encore plus évidente dans garnir( $r$ ) " approvisionner " $\left(9^{\circ}\right)$ qui concerne en premier lieu le ravitaillement (militaire et autre) et, de façon plus générale, sous $\left(10^{\circ}\right)$ « pourvu de (biens, etc.) » et sous $\left(11^{\circ}\right)$ " équiper, pourvoir 
du matériel nécessaire (p.ex. un bateau, un château, etc.) ", d'où garni de " en possession de \#. Des sens $9^{\circ}$ à $11^{\circ}$ se dégage facilement garni "bien pourvu će biens matériels = riche » $\left(12^{\circ}\right)$ qui devient épithète stéréotype d'un pays ou d'une ville. Le sens de " pourvoir ${ }^{\circ}$ peut, en c'épassant le monde matériel, concerner de même une qualité physique, morale ou intellectuelle $\left(13^{\circ}\right)$. Il peut de même se spécialiser dans certaines directions, telles " pourvoir de vêtements » (garni « vêtu » $14^{\circ}$; cp. "armé » $6^{\circ}$ ) ou « pourvoir d'accessoires » (garnir "orner » et garni "complété " $\left(15^{\circ}\right)$, sens qui annonce céjà le nouveau centre de gravité du verbe garnir en $\mathrm{mfr}$. et en frm. D'autres spécialisations du sens "pourvoir de " se trouvent de façon sporadique dans le domaine religieux avec valeur d'euphémisme (garnie « enceinte " en parlant de la Vierge $16^{\circ}$, sens d'ailleurs encore vivant, de façon beaucoup moins spiritualisée, Cans certains patois) et, dans le domaine juridique et acministratif $\left(17^{\circ}\right.$ " corroborer un acte par l'adjonction d'un sceau " [cp. garnment $11^{\circ}$; garnissement $5^{\circ}$; garnison $14^{\circ}$; congarni]; $18^{\circ}$ garnir la main de justice a consigner une somme pour assurer les intérêts du parti adverse »). Il va sans dire que le linguiste, en séparant ainsi une série de sens différents qui, souvent, ne concernent que des nuances, doit être plus rigoureux que la langue elle-même et que le lecteur doit, après avoir suivi l'analyse, reconstituer l'unité. Voici deux exemples qui serviront à mettre en relief cette unité ou ces interférences : Puis l'a d'humes garni $\left(5^{\circ} e\right.$ d'armes $\left(11^{\circ}\right)$ e de blé $\left(9^{\circ}\right)$ dans le Roman de Rou (II 1883) et Dès qu'il nos trovera guarniz, De son aguait e de ses fiz, Il n'en fera ja autre rien dans le Roman le Troie (BenTroieC 24761), où se rencontrent les sens "averti, prémuni contre un danger * $\left(1^{\circ}\right)$, "informé " $\left(3^{\circ}\right)$ et "protégé » $\left(4^{\circ} ;\right.$ le gloss. définit "garanti contre ". - La même famille se trouve Gans d'autres parties de la Romania : aesp. guarnir "amonestar, advertir (contra peligro o amenaza) ; proveer, guarnecer, armar 》 (Cid, etc., d'après Corominas 2,818 « frecuente y popular "; d'où guarnecer dep. ca.1400), aport. guarnir (mlt. 1253 : garnire scutum; sed obraduras garnescant de illis Mach 2; port. 14e s. : eu vos guarni da melhor espada; remplacé par port. guarnecer), cat. guarnir * fornir una cosa d'aquelles peces o elements que la completen o que serveixen per a la seva defensa, protecció, adorn, etc." (G̈ep. ca.1272, AlcM), sursilv. garnir " orner " VieliDec, eng. id. Peer, it. guarnire " orner ; fournir, munir, armer, équiper (milit.) ; meubler ; etc. " (dep. $13^{\mathrm{e}} \mathrm{s}$., BattAl ; mlt. guarnire en Italic v. $D C$ 4,126), roum. garnisí " guarnecer; contagiar, passar una enfermedad (arg.)" Cioranescu 3602 (emprunt prob. plutôt récent). Corominas pense que le mot germanique a été conféré au lt. vulg. par des soldats faisant service dans l'armée romaine. Mais la vitalité Ge la famille en afr. avec toutes ses ramifications sémantiques et ses dérivés et le fait que la France du Nord a été un foyer d'expansion (et ceci en premier lieu pour des termes militaires) depuis le royaume carolingien me fait accepter plutôt l'avis de Gam ${ }^{2}$ que le mot a été apporté par les Francs. La première attestation en roman se trouve dans un capitulaire de Charles le Chauve (840-877) : Unusquisque infra patriam cum pace et sine oppressione pauperum... et in hostem, vel ad placitum, sive ad curtem veniens, de suo sit warnitus ("pour$\mathrm{vu}$, muni, équipé $"$ ), et de domo sua moveat, ut cum pace venire... possit (DC $4,36 \mathrm{c})$; nobis in adjutorium, prout citius potuerint, veniant, et ad hoc omnes semper warniti sint (ib.) ; ensuite, garnitus est attesté en 925 : Ad omnem legen examinandam, quamcumque ei Synodus indicasset, semper garnitus adesset : id est instructus et proparatus DC 4,35c. FEW 17,533a ; REW 9507; Gam ${ }^{2}$; Corom ${ }^{2}$ 2,818; BattAl.] (2e m.10 ${ }^{\mathrm{e}}$ s. garnir PassionF 112 ; AlexisPF34; WaceNicD; WaceRouH II 899;2201; III 2428; etc; ErecF 1556;5803; YvainF 318 ; ChronSMichelM; BenDucF 3675;5436; MonGuill2 C 3027;3803; etc.; PercH 2000 ; etc. ; guarnir (surtout norm.agn.) AlexisLAF 34; Rol 3676;3040; PhThCompM 3;3446; CourLouisL 1580; EneasS 1 4232;4235;7155;7612; BenTroieC 241 et passim; MarieFraisneW 152; RoisL 366; WaceRouH III 183;3374; MarieFabW; SThomGuernW 3278 ; AmbroiseP 6913 ; etc. ; GarLorr (R 6,489); ChastPereB 296 ; BueveS 2,4313; SAubA 452; AuberiT 17, agn. gwarnir MirAgn ${ }^{2} \mathrm{eK}$ 60,63; rég. guernir SThomGuernW 2529 var. ms. $P[=$ Gdf $]$; RomPast II 16,28; VoeuxEpW; SEust10P 514, gernir SThomGuernW 2529 var. ms.B ; pic. wall. lorr. warnir RobClariL 37,$25 ; 43,38 ; 74,32 ; 75,2 ; 44,35 ; 61,2 ;$ doc. 1277 CPont 636 ; cioc. Liège 1287 CoutBelg V,1,409,13; doc. lorr. $1313 \mathrm{Gdf}$, agn (?) id. doc. Douai $13^{\mathrm{e}}$ (?) [charte du roi d'Angleterre ], lorr. wairnir doc. 1214 Gdfc; gairni ColMusB 2 IV 2.8 [ms. lorrain C], carni p.p. BaudSebB 455) $1^{\circ}$ garnir (qn) contre (qch.) «AVERTIR 
TABLEAU SYNOPTIQUE DES SIGNIFICATIONS DE LA FAMILLE DE GARNIR

\begin{tabular}{|c|c|c|c|c|c|c|c|c|c|c|}
\hline & garnir & attest. & $\begin{array}{l}\text { garnement } \\
\text { garniment }\end{array}$ & $\begin{array}{l}\text { garnis- } \\
\text { sement }\end{array}$ & garnisseur & $\begin{array}{c}\text { guerni- } \\
\text { sance }\end{array}$ & garnissure & garnison & $\begin{array}{l}\text { garni- } \\
\text { sonette }\end{array}$ & $\begin{array}{l}\text { garni- } \\
\text { soisson }\end{array}$ \\
\hline $1^{\circ}$ & 《avertir (d'un danger» & 34 & & & & & & & & \\
\hline $2^{\circ}$ & $\begin{array}{l}\text { « exhorter (qu à faire } \\
\text { qch) } » \leftarrow \text { angl. }\end{array}$ & 3 & . & & & & & . & & \\
\hline $3^{\circ}$ & 《instruire, informer $》$ & 26 & & $\begin{array}{l}\rightarrow 1^{\circ} \ll \text { ins- } \\
\text { truc- } \\
\text { tion } » 2\end{array}$ & & & & & & \\
\hline $4^{\circ}$ & $\begin{array}{l}\text { se tenir sur ses } \\
\text { gardes } \gg\left(\rightarrow 1^{\circ}\right)\end{array}$ & 27 & $\begin{array}{l}\rightarrow 1^{\circ} \text { défen- } \\
\text { se, pro- } \\
\text { tection } 2\end{array}$ & $\begin{array}{l}\rightarrow 2^{\circ} \ll \text { pré- } \\
\text { caution } 》 1 \\
\overrightarrow{3}^{\circ} \ll \text { pro- } \\
\text { tection } \gg 3\end{array}$ & & & & $\begin{array}{l}\rightarrow 1^{\circ} \text { 《 dé- } \\
\text { fense, pro- } \\
\text { tection } » 2\end{array}$ & & \\
\hline $5^{\circ}$ & $\begin{array}{l}\text { «occuper, faire occuper un } \\
\text { lieu pour le défendre }\end{array}$ & 88 & $\begin{array}{l}\rightarrow 2^{\circ} \text { garni- } \\
\text { son mili- } \\
\text { taire } 1 \\
\downarrow 3^{\circ} \text { guer- } \\
\text { rier } \\
\text { soldat } 2\end{array}$ & $\begin{array}{l}\rightarrow ? \ll \text { forte- } \\
\text { resse } \gg 2\end{array}$ & & & & 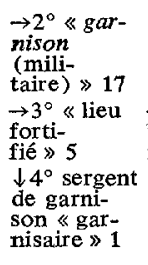 & $\begin{array}{l}\rightarrow \ll \text { petite } \\
\text { forte- } \\
\text { resse } » 1\end{array}$ & $\begin{array}{l}\rightarrow \ll \text { forte- } \\
\text { resse } \gg 2\end{array}$ \\
\hline $6^{\circ}$ & $\begin{array}{l}\text { "muni et armé de; se } \\
\text { préparer pour le combat» }\end{array}$ & 37 & $\begin{array}{l}\rightarrow 4^{\circ} \ll e q u i- \\
\text { pement } \\
\text { d'un soldat, } \\
\text { armes } 72\end{array}$ & & & & & $\begin{array}{l}\rightarrow 5^{\circ} \ll \text { pré- } \\
\text { parer } 2 \\
\text { (combat, } \\
\text { fête) } \gg\end{array}$ & & \\
\hline $7^{\circ}$ & $\begin{array}{l}\text { «se disposer à, } \\
\text { se préparer à }\left(\rightarrow 5^{\circ}\right)\end{array}$ & $5\}$ & & & & & & & & \\
\hline $8^{\circ}$ & «prêt à » & $27 J$ & & & & & & & & \\
\hline $9^{\circ}$ & «approvisionner» & 39 & & & & & & $\begin{array}{l}\rightarrow 6^{\circ} \\
\text { « equipe- } \\
\text { ment } \gg 10\end{array}$ & & \\
\hline $10^{\circ}$ & $\begin{array}{l}\text { "pourvu de (biens, etc.), } \\
\text { approvisionnê } »\end{array}$ & 19 & $\begin{array}{l}\stackrel{\downarrow}{\rightarrow} 5^{\circ} \text { équi- } \\
\text { pement } \\
\text { non mili- } \\
\text { taire } 12\end{array}$ & $\begin{array}{l}\rightarrow 4^{\circ} \\
\text { «equi- } \\
\text { pement } \\
\text { (milit.), } \\
\text { provi- } \\
\text { sions } \$ 2\end{array}$ & & $\begin{array}{l}\text { « équipe- } \\
\text { ment, ap- } \\
\text { provision- } \\
\text { nement } 1\end{array}$ & $\begin{array}{l}\rightarrow 1^{\circ} \\
\text { «équipe- } \\
\text { ment } 1 \\
\rightarrow 2^{\circ} \ll \text { biens } \\
\text { meu- } \\
\text { bles } 1\end{array}$ & $\begin{array}{l}\rightarrow 7^{\circ} \ll a p- \\
\text { provi- } \\
\text { sionne- } \\
\text { ment } \geqslant 16\end{array}$ & & \\
\hline $11^{\circ}$ & $\begin{array}{l}\text { «équiper, pourvoir du } \\
\text { matériel necessaire } \\
\text { (p.ex. un bateau, } \\
\text { un château) » } \\
\text { « en possession de }\end{array}$ & 16 & $\begin{array}{l}6^{\circ} \text { objets } \\
\text { précieux, } \\
\text { ornement, } \\
\text { parure } 8\end{array}$ & & & & & & & \\
\hline $12^{\circ}$ & \& riche $(+$ épithète $)$ & 61 & & & & & & $\begin{array}{l}\downarrow \\
\rightarrow 8^{\circ} \ll \text { bien, } \\
\text { richesse } 1 \\
6^{\circ} 7^{\circ} \\
9^{\circ} \ll \text { ré- } \\
\text { serve, } \\
\text { stock» } 15\end{array}$ & & \\
\hline $13^{\circ}$ & $\begin{array}{l}\text { «pourvu (d'une qualité } \\
\text { physique, morale ou intell.)» }\end{array}$ & 13 & & & & & & & & \\
\hline $14^{\circ}$ & $\begin{array}{l}\text { 《pourvoir de vêtements; } \\
\text { vêtu 》 }\end{array}$ & 4 & $\begin{array}{l}\rightarrow 7^{\circ} \text { vête- } \\
\text { ment } 59\end{array}$ & & & & & & & \\
\hline $15^{\circ}$ & $\begin{array}{l}\text { "pourvoir d'accessoires, } \\
\text { orner 》 }\end{array}$ & 21 & $\begin{array}{l}\downarrow \\
\rightarrow 8^{\circ} \text { pièce } \\
\text { de vête- } \\
\text { ment } 14 \\
\downarrow 9^{\circ} \ll v a u- \\
\text { rien } 1^{\circ}\end{array}$ & & $\begin{array}{l}\rightarrow \ll \text { artisan } \\
\text { qui } \\
\text { garnit } » 10\end{array}$ & & $\begin{array}{l}\rightarrow 3^{\circ} \ll \text { or- } \\
\text { nement, } \\
\text { garni- } \\
\text { ture } » 2\end{array}$ & $\begin{array}{l}\rightarrow 10^{\circ} \ll \text { or- } \\
\text { nement } 3 \\
\rightarrow 11^{\circ} \\
\ll \text { dou- } \\
\text { blure } \gg 2 \\
6^{\circ} \\
12^{\circ} \ll \text { grée- } \\
\text { ment } 1\end{array}$ & & \\
\hline $16^{\circ}$ & «enceinte (rel.)» & 1 & $\begin{array}{l}10^{\circ} \ll \text { par- } \\
\text { ties geni- } \\
\text { tales de } \\
\text { 1'homme } \\
\downarrow\left(4^{\circ} / 5^{\circ}\right) 1\end{array}$ & & & & & $\begin{array}{l}6^{\circ}\left(10^{\circ}\right) \\
13^{\circ} \text { \& par- } \\
\text { ties geni- } \\
\text { tales de } \\
\text { l'homme }\end{array}$ & & \\
\hline $17^{\circ}$ & $\begin{array}{l}\text { \&corroborer un acte par } \\
\text { l'adjonction d'un sceau } \\
\text { (jur.) })\end{array}$ & 2 & $\begin{array}{l}\rightarrow 11^{\circ} \text { ap- } \\
\text { position } \\
\text { d'un } 5 \\
\text { sceau } 5\end{array}$ & $\begin{array}{l}\rightarrow \ll \text { appo- } \\
\text { sition } \\
\text { (d'un } \\
\text { sceau) } \gg 19\end{array}$ & & & & $\begin{array}{l}14^{\circ} \text { 《ga- } \\
\text { rantie (par } \\
\text { l'apposi- } \\
\text { tion d'un } \\
\text { sceau) } \gg 1\end{array}$ & & \\
\hline $18^{\circ}$ & $\underset{\text { (jur.) })}{\operatorname{ggarnir} \text { la main de justice }}$ & $1(1318)$ & & & & & & & & \\
\hline
\end{tabular}


TABLEAU SYNOPTIQUE DES SIGNIFICATIONS DE LA FAMILLE DE GARNIR (suite)

\begin{tabular}{|c|c|c|c|c|c|c|c|c|c|c|c|}
\hline garnesture & $\begin{array}{l}\text { garneture } \\
\text { garniture }\end{array}$ & guarnerie & garnil & regarnir & engarnir & congarni & esgarni & desgarnir & $\begin{array}{c}\text { desgarnis- } \\
\text { sement }\end{array}$ & $\begin{array}{l}\text { contre- } \\
\text { garnir }\end{array}$ & $\begin{array}{l}\text { contre- } \\
\text { garnison }\end{array}$ \\
\hline & & & & & $\begin{array}{l}\rightarrow 1^{\circ} \text { \& aver- } \\
\text { ti (d'un } \\
\text { danger) } » 1\end{array}$ & & & & & & \\
\hline $\begin{array}{l}\rightarrow 1^{\circ} \text { \& pro- } \\
\text { tection (pal } \\
\text { Dieu) } \gg 1\end{array}$ & & & & & & & & $\begin{array}{l}\rightarrow 1^{\circ} \text { dé- } \\
\text { pourvu de } \\
\text { moyens de } \\
\text { défense } 14 \\
\left(5^{\circ} \rightarrow ;\right. \\
+6^{\circ} \rightarrow \\
\left.11^{\circ} \rightarrow\right)\end{array}$ & & $\begin{array}{l}\rightarrow \ll \text { prendre } \\
\text { à son tour } \\
\text { des mesu- } \\
\text { res de } \\
\text { sécurité } » 1\end{array}$ & $\begin{array}{l}\rightarrow \text { \& action } \\
\text { qu'on } \\
\text { entreprend } \\
\text { pour se } \\
\text { préserver } \\
\text { d'une } \\
\text { attaque } \gg 1\end{array}$ \\
\hline $\begin{array}{l}\rightarrow 2^{\circ} \text { "gar- } \\
\text { nison (mi- } \\
\text { litaire) } \gg 1\end{array}$ & & & & $\begin{array}{l}\rightarrow 1^{\circ} \ll \text { re- } \\
\text { mettre une } \\
\text { garnison } \\
\text { (dans une }\end{array}$ & $\begin{array}{l}\rightarrow 2^{\circ} \ll \text { occu- } \\
\text { per une } \\
\text { place pour } \\
\text { la defen- }\end{array}$ & & & $\begin{array}{l}\rightarrow 2^{\circ} \ll \text { aban- } \\
\text { donner } \\
\text { quitter } \\
\text { (une }\end{array}$ & & & \\
\hline $\begin{array}{l}\rightarrow 3^{\circ} \ll \text { for- } \\
\text { teresse } » 1 \\
{[+\& \text { équi- }} \\
\text { pement mi- } \\
\text { litaire »? 1] }\end{array}$ & & & & $\begin{array}{l}\text { ville, } \\
\text { etc. }) » 1 \\
\rightarrow 2^{\circ} \ll \text { re- } \\
\text { mettre en } \\
\text { état de } \\
\text { défense } » 2 \\
\left(+10^{\circ} \rightarrow\right. \\
\left.11^{\circ} \rightarrow\right)\end{array}$ & dre $\gg 1$ & & & terre) $\gg 1$ & & & \\
\hline & & & aoccit. & $\begin{array}{l}3^{\circ} \text { « remet- } \\
\text { tre en état } \\
\text { (des engins } \\
\text { de } \\
\text { guerre) } \gg 1\end{array}$ & & & $\begin{array}{l}\rightarrow \text { « dépour- } \\
\text { vu } \\
\text { d'armes » } 1\end{array}$ & $\begin{array}{l}\rightarrow \text { «e dé } \\
\text { pouiller de } \\
\text { ses armes } \\
\left(\text { sub } 1^{\circ} \text { ) } 3\right. \\
\rightarrow 3^{\circ} \text { 《ne } \\
\text { pas prêt } \\
\text { de } » 1\end{array}$ & & & \\
\hline & & & $\begin{array}{c}\downarrow \\
\ll \text { filet de } \\
\text { pếche 》 } 3\end{array}$ & & & & & $\overrightarrow{d e} 4^{\circ} \ll{ }_{8}$ privé & & & \\
\hline \multicolumn{12}{|l|}{$\begin{array}{l}\rightarrow 4^{\circ} \text { « équi- } \\
\text { pement, } \\
\text { surtout } \\
\text { provisions } \\
\text { alimen- } \\
\text { taires » } 11\end{array}$} \\
\hline & & & & & & & & $\begin{array}{l}\rightarrow 9^{\circ} \ll \text { sans } \\
\text { argent } \gg 2\end{array}$ & & & \\
\hline \multirow[t]{4}{*}{. } & & $\begin{array}{l}\overrightarrow{\vec{s}} \ll \operatorname{maga}- \\
\text { sin, entre- } \\
\text { pôt } \gg 1\end{array}$ & & & & & & & & & \\
\hline & & & & & & & & $\begin{array}{l}\rightarrow 6^{\circ} \ll \text { pri- } \\
\text { vé dé- } \\
\text { pourvu de } \\
\text { sens } \gg 2\end{array}$ & 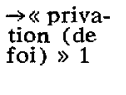 & & \\
\hline & $\begin{array}{l}\rightarrow \ll \text { orne- } \\
\text { ment, } \\
\text { accessoi- } \\
\text { res } \gg 2\end{array}$ & & & & & & & & & & \\
\hline & & & & & $\begin{array}{l}\rightarrow 3^{\circ} \ll \text { gar- } \\
\text { nir, } \\
\text { orner } \$ 1\end{array}$ & & & & & & \\
\hline $\begin{array}{l}\rightarrow 5^{\circ} \ll \text { vali- } \\
\text { dation } \\
\text { (d'un } \\
\text { acte) } \gg 1\end{array}$ & & & & & & 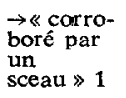 & & & & & \\
\hline
\end{tabular}


(qn de qch.), mettre en garcie, prémunir (qn contre qch.) » (PassionK 112 [A cel sopar un sermon fez (Jésus)... Contrals afanz que an apader Toz sos fidels ben en garnid], TL 4,186; GdfC 9,687b; FEW 17,529a, $\square$ garnir (qn contre qch.) « avertir (qn d'un danger, etc.) 》 EneasS 1 4232;4235; BenTroieC 27961 [Mout vos manace, e si a dreit. A escient vos en guarnis : Guardez tel conseil aiez pris Par que ne seiez engeigniee Ne de vostre terre chaciee! $R 42,99$ corrige le gloss. de Constans qui céfinit " affirmer qch. à qn "] ; ErecF 5803 ; RouH II 2201 ; MarieFabW 70,10 [si parent e si ami L'en aveient [...]

\section{Le statut des dérivés}

Ce tableau montre en même temps quatre autres faits essentiels valables pour les dictionnaires diachroniques ou génétiques :

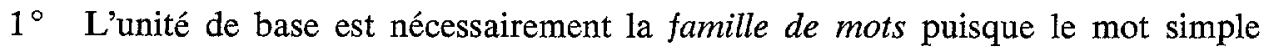
est en rapport étroit avec ses dérivés. Les dérivés se raccrochent à certains sens du mot de base. Le jeu de ces « raccrochages » est de première importance pour la fonction des préfixes et des suffixes. Les influences semblent d'ailleurs jouer dans les deux sens (v. p. ex. garnement « vêtement 》 beaucoup plus fréquent que garnir «pouvoir de vêtements » et garni «vêtu»), bien que la direction mot de base $\rightarrow$ dérivé semble être de beaucoup la plus fréquente, c'est-à-dire normale.

$2^{\circ}$ ) Le statut génétique des dérivés (et dans certains cas celui des mots simples, v. ci-dessus) est complexe. Ils sont engagés dans deux directions : horizontalement, ils dépendent génétiquement du mot de base (c'est le statut le plus fréquent, c'est-à-dire normal, du moins dans le cas des dérivés de garnir) ; verticalement, certains sens s'expliquent génétiquement par d'autres sens, d'un même dérivé (v. p. ex. garnement $9^{\circ}$ «vaurien $\left.\gg\right)$. Dans certains cas, les rapports génétiques sont ambigus; il y a convergence de rapports horizontaux et verticaux (dont même la linguistique, avide de solutions univoques, doit tenir compte !).

$3^{\circ}$ ) Le dictionnaire génétique, linéaire par la force des choses, doit opter pour une organisation horizontale (classement onomasiologique à travers toute la famille de mots : en principe, système du FEW) ou pour une organisation verticale (classement sémasiologique laissant intacts les ensembles de champs sémasiologiques : système du DEAF). Un choix entre les deux est inévitable et relève de la bipolarité du signe linguistique en tant que signe constitué par une forme de l'expression et une forme du contenu. On se trouve devant une décision élémentaire entre la présentation sémasiologique coupant les relations onomasiologiques (DEAF) et la présentation onomasiologique coupant les relations sémasiologiques (FEW ; dans certains cas, d'ailleurs, surtout dans les articles difficiles à structurer, le FEW, lui aussi, a choisi la première méthode). Nous avons choisi la méthode sémasiologique pour le DEAF parce qu'elle est toujours applicable et parce qu'il est plus facile de rétablir les rapports onomasiologiques par des renvois. La méthode onomasiologique, appliquée de façon rigoureuse, aurait pour conséquence un morcellement considérable de la famille; le FEW procède pour cette raison par groupes onomasiologiques; il choisit donc, en réalité, une solution intermédiaire entre les deux principes (v. p. ex. l'article *WARDÔN $(17,510)$, où Wartburg distingue deux groupes : «betrachten ( « regarder $)$ et «bewachen » («garder »), c'est-à-dire les deux fonctions de base qui expliquent les 27 (!) significations du DEAF). 
$4^{\circ}$ ) Le statut génétique ambigu des dérivés que nous venons de décrire a des conséquences pour le classement des sens des dérivés. Il va sans dire que les rapports génétiques verticaux (ils concernent les sens qui s'expliquent à l'intérieur même de l'évolution sémantique du dérivé) suivent la règle générale de l'organisation génétique des significations. Mais garnir nous montre que c'est plutôt une exception. Si les rapports génétiques horizontaux, qui relient certaines significations du dérivé à certaines significations du mot de base, sont plus fréquents, comme dans le cas de la famille de garnir, il y a avantage à respecter le classement établi pour le mot de base, même si la chronologie des significations du dérivé ne s'accorde pas avec ce classement. Puisque ces significations dépendent uniquement $\mathrm{du}$ mot de base, et non d'une évolution interne, la chronologie devient, en effet, purement fortuite. Les adverbes le prouvent d'ailleurs de façon évidente : à chaque moment de l'histoire de l'adj. de base, il est possible de former un adverbe correspondant à chacune de ses significations (s'il n'y a pas de restrictions pour d'autres raisons); il y a donc une série d'actes créateurs successifs qui, verticalement, peuvent être indépendants l'un de l'autre ${ }^{30}$. Le classement des significations des dérivés devra donc suivre, en principe, le classement du mot de base (relations horizontales), dans lequel on intercalera les significations de genèse interne (relations verticales).

\section{Le statut du mot de base}

Le classement du mot de base doit suivre uniquement le principe génétique, nous l'avons vu. Là, contrairement au statut des dérivés, la filiation des sens est purement interne (sauf dans les cas de réaction d'un dérivé sur le mot de base, ce qui semble être plutôt rare). Comme la filiation des sens est rarement linéaire, il faudra recourir à des flèches indiquant les rapports génétiques :

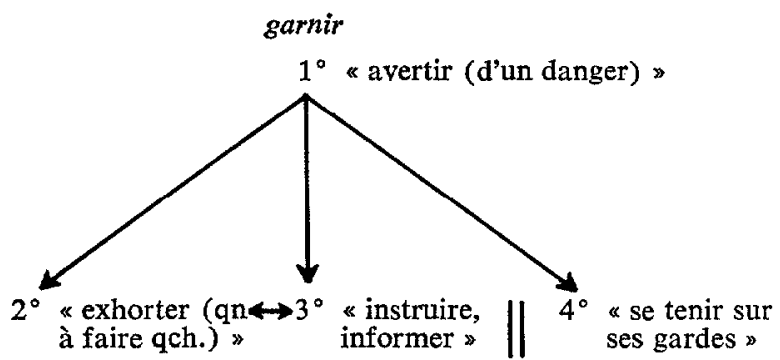

Dans le dictionnaire, ces rapports se présenteront de la façon suivante : $1^{\circ} \ldots 2^{\circ} \ldots 3^{\circ} \ldots 4^{\circ}\left(\rightarrow 1^{\circ}\right)$. Nous renonçons à faire des renvois sous $3^{\circ}$ parce qu'il peut y avoir des relations génétiques aussi bien entre $1^{\circ}$ et $3^{\circ}$ qu'entre $2^{\circ}$ et $3^{\circ}$. Les flèches ne pourront signaler que les bifurcations principales. Le jeu des relations est souvent très complexe, v. l'introduction à l'article garnir.

30. Les adverbes (plus encore que les autres dérivés) sont souvent très mal analysés dans les dictionnaires. Ceux-ci se contentent souvent de définir « d'une manière + adj. de base ", ce qui établit un lien horizontal sans préciser les significations du mot de base réellement concernées. 
Une chose est certaine : la filiation des sens suivra de préférence un principe sémantique. Cela distinguera nettement le FEW et le DEAF d'un dictionnaire philologique tel que le TL. Le dictionnaire génétique est soumis à une classification sémantique, le dictionnaire philologique, à une classification grammaticale. Comparons les articles gaires dans le TL et le DEAF :

TL

[1. ] beaucoup, très; longtemps (avec ne en phrase nég.)

[2. ] avec senz

[3. ] (dans des phrases de contenu négatif)

[4. ] ne... faire sans verbe

[5. ] ne gaire à côté d'un verbe + nég.

[6. ] n'a faire (frm. naguère)

[7. ] n'a gaire(s) que * il n'y a pas longtemps que $\gg$

[8. ] n'a gaire(s) «cela arrivera en peu de temps 2

[9. ] ne... de gaire(s) " pas beaucoup, guère 》

[10.] gaire(s) dans une phrase interrog. pos. : beaucoup (sehr viel)

\section{DEAF}

1. ne... gaires «pas très ( + adv., adj.) ", " pas beaucoup ( + v., subst.) »

$\rightarrow$ gaires ( + adj., adv.) "très », gaires de ( + subst. $)$ « beaucoup » TL $1,2,3,5,9,10$ TL $1,2,3,5,9,10$

2. ne... guaires de tens "pas longtemps", ne... gaires, " id. "

$\rightarrow$ gaires «longtemps»

TL 1,3

3. *ainz ne gaires « bientôt, d'ici peu » TL 4

4. jusqu'a ne gaires, « bientôt, d'ici peu » TL 4,8

5. n'a gaires

$\rightarrow$ nagaires \& il n'y a pas longtemps "

TL 6,7

Ce tableau montre à l'évidence que les principes de classement sont tout à fait différents. On trouve sous $1^{\circ}$, dans le DEAF, des exemples classés par TL sous 1,2,3,5,9,10 (etc.). Dans les articles concernant les verbes, cette différence n'est pas moins évidente (TL classe d'après v. a, v. n., pron., p. p., etc., tandis que, dans le DEAF, ces différentes réalisations grammaticales sont classées sous les significations correspondantes; cp. garder, garnir, etc.).

La différence des principes se manifeste également dans le traitement des conséquences sémantiques des emplois négatifs et positifs. Génétiquement l'emploi négatif précède et explique souvent une nouvelle signification positive :

$1^{\circ}$ ne... gaires "pas très, pas beaucoup 》 $\rightarrow$ gaires «très, beaucoup »

$2^{\circ}$ ne... gaires $\ll$ pas longtemps $\gg \rightarrow$ gaires $«$ longtemps $»$.

Nous avons classé ces inversions sémantiques sous les numéros sémantiques correspondants de l'ordre génétique. Le TL ne fait pas ressortir ce rapport génétique. Ce jeu d'inversion sémantique apparaît aussi, p. ex., sous garder (DEAF) : $5^{\circ}$ garder que + subord. pos. "veiller à ce que, faire cn sorte que $\gg$ [pos.] $\rightarrow 9^{\circ}$ garder que... ne «empêcher que » [nég. avec inversion sémantique] $\rightarrow$ soi garder de qch., de faire qch. «éviter, empêcher » [pos. avec le sens nég.]

On retrouve le même phénomène amorcé sous regarder :

regarder qch. «prendre en considération »[pos.]

$\rightarrow$ ne regarder qch. «ne pas prendre en considération $»=$ "pardonner [nég. $\rightarrow$ nouveau sens pos. par inversion sémantique] ${ }^{31}$.

31. P.ex. prïoit a Diu... Que il de honte le gardast Et qu'envers lui ne regardast Les granz pechiez qu'il avoit fais (EscanorM 7633). 
L'emploi négatif au sens de «pardonner »n'étant pas fréquent, le passage de ne regarder «pardonner » à regarder «pardonner » ne s'est pas produit.

Je n'ai pu aborder que quelques-uns (mais peut-être les plus importants) des problèmes théoriques et pratiques concernant un dictionnaire diachronique ou génétique. Il faut prendre conscience très clairement de tous ces problèmes, d'autant plus qu'il sont multiples et souvent délicats.

Kurt Baldinger 